\title{
ASSESSING THE GENETIC RELATIONSHIP OF TAIF ROSE WITH SOME ROSE GENOTYPES (ROSA SP.) BASED ON RANDOM AMPLIFIED POL YMORPHIC DNA, INTER SIMPLE SEQUENCE REPEAT AND SIMPLE SEQUENCE REPEAT MARKERS
}

\author{
${ }^{1}$ Salah El-Din El-Assal, ${ }^{1,2}$ Mohamed A. El-Awady, ${ }^{1,2}$ Adel El-Tarras and ${ }^{3,4}$ Gaber Shehab \\ ${ }^{1}$ Department of Genetics, Faculty of Agriculture, Cairo University, El-Gamaa Street, P.O. Box 12613 Giza, Egypt \\ ${ }^{2}$ Biotechnology and Genetic Engineering Unit, Deanship of Scientific Research, Taif University, Hawya, KSA \\ ${ }^{3}$ Department of Agricultural Chemistry, Faculty of Agriculture, Cairo University, El-Gamaa Street, P.O. Box 12613 Giza, Egypt \\ ${ }^{4}$ Faculty of Medicine, Taif University, Hawya, KSA \\ Received 2013-11-18; Revised 2014-04-26; Accepted 2014-04-29
}

\begin{abstract}
Taif-roses are a famous rose type that cultivated in Taif region and well known with their deep and intensive fragrance in the Arabian World. Despite of the great economical importance of Taif-roses for the kingdom of Saudi Arabia, their genetic origin has not been yet elucidated. The present study was mainly aimed to assess the genetic relationship between Taif-roses and some rose genotypes that grown in some kingdom neighboring countries using molecular markers and aromatic amino acids contents. Three Taif-roses genotypes namely Hada, Shafa-1 and Shafa-2 were compared to nine different rose genotypes that are grown in Egypt and Syria. Out of 12 RAPD, 8 ISSR and 8 SSR primers used, clear and repeatable band profile of 8, 6 and 7 primers was obtained from the three markers, respectively. Total of 111, 64 and 15 bands with polymorohism of 96.4, 90.6 and $93.3 \%$ were obtained using RAPD, ISSR and SSR, respectively. The discriminating power of the three markers has led to efficient grouping of the 12 rose genotypes using Unweighted Pair Group Method (UPGMA). Among the 12 genotypes, Syrian-Gory rose shown the highest genetic similarity of 75, 92 and $65 \%$ with the three Taif-roses genotypes Hada, Shafa-1 and Shafa-2, respectively. The established dendrogram was clearly separated the 12 rose genotypes into four major groups in which the three Taif-roses genotypes were clustered in the same group with the Gory rose-Syrian genotype. Moreover, the data revealed that among the studied rose genotypes, the contents of aromatic amino acids in Syrian-Gory rose and the Taif rose-Hada was the highest and followed by the Egyptian Balady rose 1. While Dutch rose 1, 2, 3 and Dutch tulip 1, 2 were recorded to be the lowest. Together, these results indicate that Taif-rose has closed genetic relations to the Gory rose-Syrian cultivated in Syria. Additionally, a reproducible protocol for In vitro propagation, of Taif-rose genotype (Hada) was developed.
\end{abstract}

Keywords: Taif Roses, Genetic Relationship, RAPD, ISSR, SSR, Amino Acids Analysis and Regeneration

\section{INTRODUCTION}

The roses of Al-Taif are well known with their deep and intensive fragrance in the Arabian region. These oilrich, 30-petal rose has been cultivated in Taif for three centuries. Taif city is characterized with its favorable temperatures (as West of the Taif city rises above 2000 meters over the see level), plentiful groundwater, wellestablished irrigation systems and fine topsoil. These advantages have combined to earn the region the name Corresponding Author: Salah El-Din El-Assal, Department of Genetics, Faculty of Agriculture, Cairo University, El-Gamaa Street, P.O. Box 12613 Giza, Egypt 
of "Arabia's Rose," ever since roses began to be cultivated there in the Ottoman era. Taif-roses have an important role in the national economy for their great value in exportation of their oil to the Arab countries, ornamentation, medicinal use as well as manufacture of perfumes (Bazaid, 2004). The flowers, whose fragrance is even more intensive than fragrance of the Damask Rose, are harvested in April. Despite of the likely origin of El Taif-Roses as a Damask Rose (Rosa x Damascena trigintipetala), it has never been completely cleared how it appeared in Taif. However, due to their close resemblance famous Bulgarian "kazanlik" strain, it has been suggested that Taif-Roses were brought from Balkans by Turks, who occupied this area in the 14th century. The genetic relations of the Taif-Roses with other rose genotypes have never been studies before. In addition, production of Taif-Roses is modest when compared to the quantities produced by larger and export-oriented operations countries such as Turkey, Bulgaria, Russia, China, India, Morocco and Iran.

DNA-based molecular marker systems are efficient and informative for genetic analysis of roses because DNA polymorphism indicated by these markers is not affected by environmental conditions. Several molecular marker systems, i.e., Restriction Fragment Length Polymorphism (RFLP) (Rajapakse et al., 2001), Random Amplified Polymorphic DNA (RAPD) (Atienza et al., 2005; Kaur et al., 2007) Simple Sequence Repeat (SSR) (Esselink et al., 2003) and Amplified Fragment Length Polymorphism (AFLP) (Baydar et al., 2004) have been used for genetic analysis of rose species, cultivars and rootstocks. RAPD markers have been widely used in many plant species such as anthurium (Nowbuth et al., 2005), strawberry (Gaafar and Saker, 2006), polyscias and schefflera (Rout et al., 2007), banana (Brown et al., 2009), olive (Sesli and Yeğenoğlu, 2009) for identification, varietal analysis, population studies and genetic linkage mapping. RAPD markers are used to detect DNA polymorphism without requirement of previous knowledge of the target genome (Williams et al., 1990). Microsatellite markers are also markers of choice (Esselink et al., 2003) because they are abundant, uniformly distributed, highly polymorphic, codominant, rapidly produced by PCR and easily accessed through published primer sequences (Gupta and Varshney, 2000).

The major objective of this study was to use the molecular markers RAPD, ISSR and SSR to investigate the genetic relations of Taif-Roses with some rose genotypes that are cultivating in some kingdom-borders countries. Also the total amino acid and aromatic components of these genotypes were measured and used for comparison. Moreover, the development of simple micropropagation system for mass production of Taifroses was also addressed.

\section{MATERIALS AND METHODS}

\subsection{Materials}

Three genotypes of Taif-Roses grown in Taif city in the Kingdome of Saudi Arabia namely Hada, Shafa-1 and Shafa-2 were collected in addition to other nine different rose genotypes that are cultivating in Egypt and Syria and used in this study. The nine rose genotypes are namely, Balady rose 1, 2 and 3 (from Egypt), Dutch rose 1, 2 and 3, Dutch Tulips 1 and 2 (cultivated in Egypt) and Gory rose-Syrian (cultivated in Syria). The stem nodes of rose plants (genotype Hada) were collected and were used for developing a micropropagation system for mass production of Taif- Roses.

\subsection{Methods}

\subsubsection{DNA Isolation}

The DNA was extracted from the 12 rose genotypes and was used as templates for 12 RAPD, 8 ISSR and 8 SSR reactions. DNA extraction at sufficient amount and quality is very difficult from rose leaves because the presence of the secondary compounds. To get rid of the phenolic compounds, we applied the protocol of Doyle and Doyle (1988) for DNA isolation using Cationic hexadecyl Trimethyl Ammonium Bromide (CTAB) with slight modifications. Two times extraction with 2mercaptoethanol was performed after the addition of an antioxidant and PVP to bound the phenolic compounds.

\subsection{RAPD Analysis}

\subsubsection{PCR Reaction and Condition}

A total of 12 random primers were used to detect the polymorphism among the 12 different rose genotypes. The sequence of the 8 primers that produce a clear and repeatable banding pattern is shown in (Table 1). The amplification was performed in a $25 \mu \mathrm{L}$ reaction volume containing about $3 \mu \mathrm{L}(10 \mathrm{ng} \mu \mathrm{L})$ genomic DNA, $3 \mu \mathrm{L}$ primer (Operon Technologies Inc.) and $19 \mu \mathrm{L}$ master mix (Promega). The PCR temperature profile was applied through a Gene Amp® PCR System 9700 (Perkin Elmer, England). The thermal cycler was programmed with an initial step of $5 \mathrm{~min}$ at $94^{\circ} \mathrm{C}$ that was followed by 40 repeated cycles of $60 \mathrm{~s}$ at $94^{\circ} \mathrm{C}$, an annealing step of $1 \mathrm{~min}$ at $37^{\circ} \mathrm{C}$ and an elongation step of $1 \mathrm{~min}$ at $72^{\circ} \mathrm{C}$; and finally a $7 \mathrm{~min}$ extension at $72^{\circ} \mathrm{C}$. The amplification 
products were resolved by electrophoresis in a $1.5 \%$ agarose gel containing ethidium bromide $(0.5 \mu \mathrm{g} \mathrm{mL})$ in $1 \mathrm{X}$ TBE buffer at 95 volts. PCR products were visualized on UV light and photographed using a gel documentation system (Bio-Rad® Gel Doc-2000).

\subsection{ISSR Analysis}

Eight ISSR primers were tested using a specific and optimal annealing temperature for each one. Only 6 ISSR primers showed repeatable products (Table 2). PCR reactions were performed in a volume of $20 \mu \mathrm{L}$ in Eppendorf thermocycler. The reaction mixture contained $0.6 \mu \mathrm{L}$ of each primer, $100 \mu \mathrm{M}$ of each deoxinucleotide, 0.5 units of Go Taq polymerase (Promega), 10x Taq buffer containing $2.5 \mathrm{mM} \mathrm{MgCl} 2$ and $10 \mathrm{ng}$ of template DNA. Amplification reaction was $94^{\circ} \mathrm{C} / 5 \mathrm{~min}$, followed by 30 cycles of $94^{\circ} \mathrm{C} / 1 \mathrm{~min}, 48-60^{\circ} \mathrm{C}$ (specific for each primer) $/ 1$ min and $72^{\circ} \mathrm{C} / 2$ min and ending with an extension step of $72^{\circ} \mathrm{C} / 7 \mathrm{~min}$. PCR products were analyzed using agarose $(2 \% \mathrm{w} / \mathrm{v})$ electrophoresis gels stained with ethidium bromide and only bands with high intensity and well separated were selected.

\subsection{SSR Analysis}

Out of 8 pair of primers that were tested to detect the polymorphism, seven pairs have recorded clear reproducible amplifications among the 12 rose genotypes (Table 3). The amplification was performed in a $25 \mu \mathrm{L}$ final volume of a reaction mixture, which included reaction buffer $1 \mathrm{x}, 0.2 \mathrm{mM}$ each deoxinucleotide, 0.25
$\mathrm{mM}$ each primer, $1.5 \mathrm{Mm} \mathrm{MgCl} 2,1 \mathrm{U}$ of enzyme Taq polymerase (Promega) and 50-100 ng genomic DNA matrix. The PCR reaction was performed using a thermo-cycler (Applied Biosystem GeneAmp 9600) programmed for: $3 \mathrm{~min}$ initial denaturation at $94^{\circ} \mathrm{C}$, followed by 35 cycles, each consisting of: $1 \mathrm{~min}$ at $94^{\circ} \mathrm{C}$, $1 \mathrm{~min}$ at $61^{\circ} \mathrm{C}, 2 \mathrm{~min}$ at $72^{\circ} \mathrm{C}$ and a final extension of 10 min at $72^{\circ} \mathrm{C}$. PCR products were evaluated by electrophoresis, on $2 \%$ agarose gels in $0.5 x$ TBE buffer, stained with ethidium bromide and were recorded as BioPrint images.

\subsection{Data Analysis}

PCR reactions were repeated three times and only reproducible bands were scored. Each polymorphic amplified fragment was scored as presence (1) and absence (0) and data were transcribed into binary format. Based on the matrix of genetic similarity, cluster analysis was done. The Unweighted Pair Group Method with Arithmetic Averages (UPGMA) method was used for clustering employing the NTSYS pc (Numerical Taxonomy and Multivariate Analysis System, version 1.8) program (Rholf, 1994).

\subsection{Amino Acid Analysis}

Different amino acids were extracted from rose petioles and analyzed using the LC-6A HPLC system (LC-6A HPLC, Shimadzu, Kyoto, Japan). The amino acidswas extracted from $1 \mathrm{gm}$ of dried rose petioles as described by (Yamanaka et al., 1994; Helsen et al., 2008).

Table 1: List of random primers that have been used for RAPD analysis

\begin{tabular}{llccr}
\hline Primer name & Primer sequence & No. of amplicons & No. of polymorphic bands & Polymorphism $(\%)$ \\
\hline OPA-04 & 5'-AATCGGGCTG-3' & 12 & 11 & 92 \\
OPA-09 & 5'-GGGTAACGCC-3' & 14 & 13 & 93 \\
OPA-15 & 5'-TTCCGAACCC-3' & 12 & 12 & 100 \\
OPA-20 & 5'-GTTGCGATCC-3' & 19 & 19 & 100 \\
OPB-03 & 5'-CATCCCCCTG-3' & 11 & 10 & 91 \\
OPB-16 & 5'-TTTGCCCGGA-3' & 21 & 21 & 100 \\
OPC-02 & 5'-GTGAGGCGTC-3' & 10 & 09 & 100 \\
OPC-07 & 5-'GTCCCGACGA-3'- & 12 & 12 & 100 \\
Total & & 111 & 107 & 96.4 \\
\hline
\end{tabular}

Table 2: List of primers that have been used for ISSR analysis (Jabbarzadeh et al., 2010)

\begin{tabular}{llllc}
\hline Primer name & Sequence & No. of Amplicons & No. of polymorphic bands & Polymorphism $(\%)$ \\
\hline ISSR 1 & 5-CCA(CT)8-3 & 11 & 11 & 100 \\
ISSR 2 & 5-(GTG)3GC-3 & 11 & 11 & 100 \\
ISSR 3 & 5-(CT)8AC-3 & 11 & 11 & 100 \\
ISSR 4 & 5-(GA)8ACC-3 & 12 & 10 & 83 \\
ISSR 5 & 5-(GT)6CC-3 & 09 & 08 & 89 \\
ISSR 6 & 5-CCA(AG)8T-3 & 10 & 07 & 70 \\
Total & & 64 & 58 & 90.6 \\
\hline
\end{tabular}


Table 3. SSR markers and corresponding primers used in this study (Zhang et al., 2006)

\begin{tabular}{|c|c|c|c|c|c|}
\hline Marker name & & Primer & No. of amplicons & No. of polymorphic bands & Polymorphism \% \\
\hline \multirow[t]{2}{*}{ Rw10J19 } & $\mathrm{F}$ & GCGAGTTGACGACGAGTT & & & \\
\hline & $\mathrm{R}$ & GGGTGGGCTTCCTTAGTTA & 3 & 3 & 100 \\
\hline \multirow[t]{2}{*}{ Rw4E22 } & $\mathrm{F}$ & ATGGGAGACAGAGGTGTAAG & & & \\
\hline & $\mathrm{R}$ & TCCTAACTCTCGGTGGAGAT & 3 & 3 & 100 \\
\hline \multirow[t]{2}{*}{ Rw3N19 } & $\mathrm{F}$ & CTGGCTGGTTCTCTTTCTG & & & \\
\hline & $\mathrm{R}$ & ATGGGTCGTCGTCGATATG & 2 & 2 & 100 \\
\hline \multirow[t]{2}{*}{ Rw17I7 } & $\mathrm{F}$ & CAGGTAATTTGCGGATGAAG & & & \\
\hline & $\mathrm{R}$ & GATCCGCCGTTTCCAGT & 2 & 2 & 100 \\
\hline \multirow[t]{2}{*}{ Rw14A5 } & $\mathrm{F}$ & СССТСААААССССТСТТА & & & \\
\hline & $\mathrm{R}$ & CGTAATAACGGTAATAAAATC & 2 & 2 & 100 \\
\hline \multirow[t]{2}{*}{ Rw22A3 } & $\mathrm{F}$ & AGAGAATTGAAAAGGGCAAG & & & \\
\hline & $\mathrm{R}$ & GAGCAAGCAAGACACTGTAA & 2 & 2 & 100 \\
\hline \multirow[t]{2}{*}{ Rw22B6 } & $\mathrm{F}$ & ACAGTGAGTTGTTCGCTTCT & & & \\
\hline & $\mathrm{R}$ & TTCATTGCTAGGAAGCAGTA & 1 & 0 & 0 \\
\hline Total & & & 15 & 14 & 93.3 \\
\hline
\end{tabular}

\subsection{In vitro Propagation of Taif-Roses}

The stem nods of the Taif-Rose genotype Hada were used for the development of micropropagation system. Nodal explants containing lateral buds of actively fieldgrown were cut and sterilized with $0.1 \%$ solution of mercuric chloride for $10 \mathrm{~min}$. The disinfected explants were inoculated aseptically on culture medium (Murashige and Skoog, 1962) supplemented with different concentration of 2,4, D (1.0 to $\left.4.0 \mathrm{mg} \mathrm{L}^{-1}\right)$ singly or in combination with Kinetin $\left(1 \mathrm{mg} \mathrm{L}^{-1}\right)$. The $\mathrm{pH}$ of medium was adjusted to 5.7 and solidified by $0.6 \mathrm{~g}$ $\mathrm{L}^{-1}$ gelan gum. It was autoclaved at $121^{\circ} \mathrm{C}$ and pressure of $1 \mathrm{~kg} / \mathrm{cm}^{2}$ for $20 \mathrm{~min}$. For subsequent subcultures, BAP (1.0 to $4.0 \mathrm{mg} \mathrm{L}^{-1}$ ) were added to the basal medium for induction of multiple shoots. Each treatment was consisted of 3 replications in which 10 to 12 explants were used. The LSD analysis was performed using the Analyse-it software LTD (PO box 77, Leeds, LS125XA, UK) according to Maxwell and Delaney (1989). All cultures were grown under a photoperiod of $16 \mathrm{~h}$ light from white fluorescent tubes at a temperature of $25 \pm 1^{\circ} \mathrm{C}$.

\section{RESULTS}

\subsection{RAPD-PCR Fingerprinting of Rose Genotypes}

Out of tested 12 RAPD primers only eight repeatable reactions were reproducible. The RAPD profiles obtained by primers OPA-04, OPA-09 and OPC-02 are illustrated in Fig. 1. PCR products of these 8 primers were used for analyzing genetic relations of Taif-Roses with other 9 rose genotypes. Amplified bands information related to each primer is giving in Table $\mathbf{1}$. Total number of amplified products was 111 bands with molecular weight ranging from 100 to 2500 bp with $96.4 \%$ of total polymorphism. The RAPD-PCR reaction with the primer OPA-16 produced the highest numbers of band (21), while the lowest (10) was obtained with the primer OPC-02 (Fig. 1). Three primers namely OPA-O4, OPA-O9 and OPB-03 showed 92, 93 and $91 \%$ of polymorphism, respectively, while the other six primers showed $100 \%$ polymorphism. The average of amplified and polymorphic bands per each primer was 13.8 and 13.4 , respectively.

\subsection{ISSR-PCR Fingerprinting of Rose Genotypes}

Six ISSR reactions revealed 64 amplified products ranging from 450 to $2500 \mathrm{bp}$ in which 66 (90.6) were polymorphic. The ISSR profiles obtained by some ISSR primers are shown in Fig. 2. Maximum numbers of bands was generated by ISSR 4 (12 bands with $83 \%$ polymorphic), while, the minimum numbers of bands was generated by ISSR 5 (9 bands with $89 \%$ polymorphic), respectively. The average of amplified and polymorphic bands per each primer was 10.7 and 9.6, respectively. Amplified bands information related to each primer is giving in Table 2. The studied primer sequences were composed of di and tri-nucleotide repeat sequences. The highest polymorphism observed in the case of tri-nucleotide repeat primers ISSR 2.

\subsection{SSR Analysis Fingerprinting of Rose Genotypes}

In the present study, out of the eight primer pairs flanking simple sequence repeats that were employed to investigate the level of polymorphism among the 12 rose genotypes, seven primers showed clear and reproducible products. 

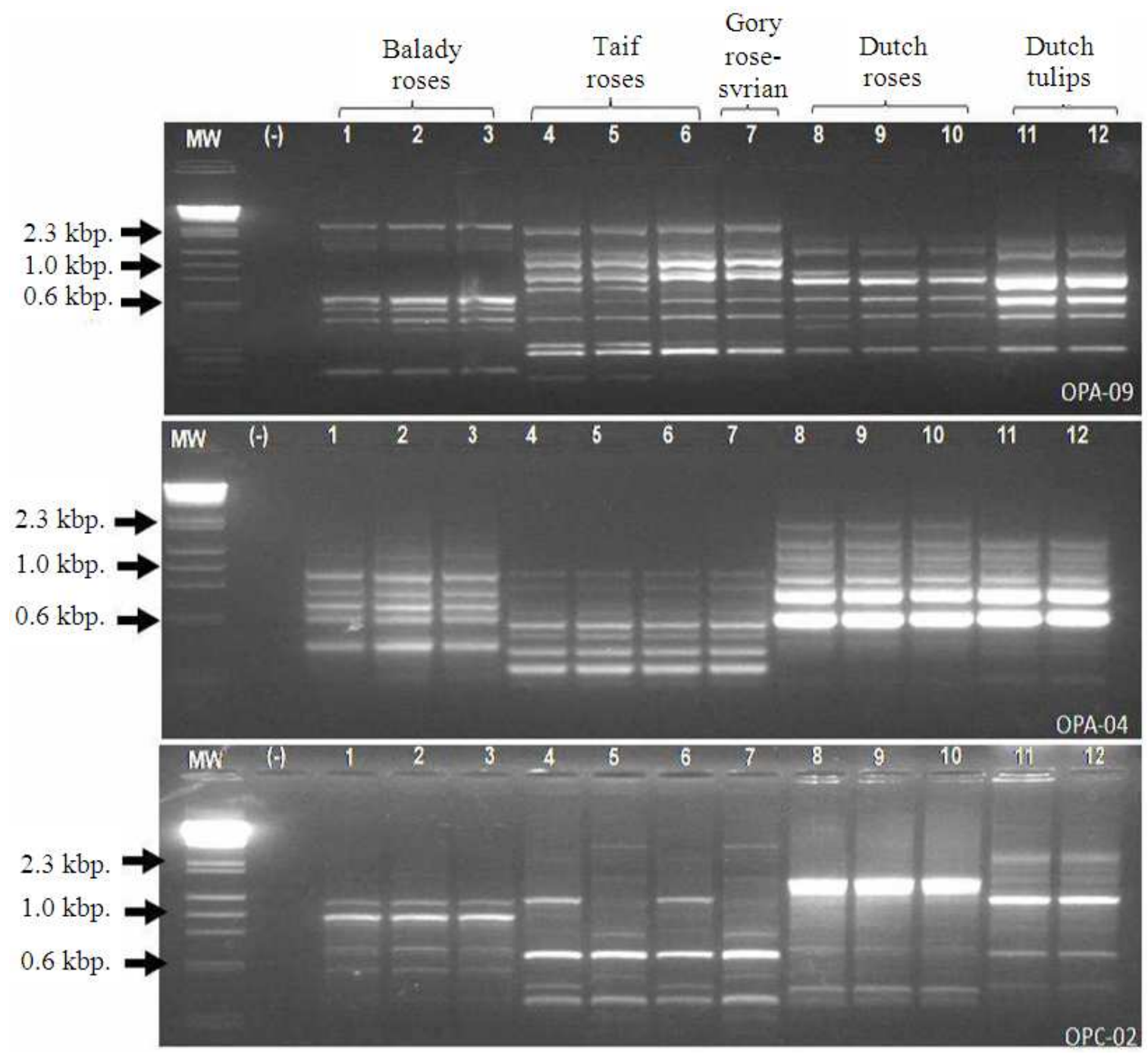

Fig. 1. RAPD profile of 12 different rose genotypes using the three primers OPA-09, OPA-04 and OPC-02. MW: is $1 \mathrm{~kb}$. Ladder; (-) is a negative PCR sample. (1-3) Balady rose1, 2 and 3 ; 4-6) Taif rose (Hada, Shafa 1 and Shafa 2; (7) Gory rose-Syrian; (810) Dutch rose1, 2 and 3 and (11-12) Dutch Tulips 1 and 2

The reproducible (true) PCR product obtained using the primers pair for the markers Rw10J19, Rw4E22 and Rw3N19 are depicted in Fig. 3. The number of alleles per marker ranged from 1 to 3 with an average of 2.1 alleles per locus (Table 3).

\subsection{Genetic Relationships as Revealed by RAPD, ISSR and SSR Markers and Cluster Analysis}

To examine the genetic relationships of Taif-Roses with other rose genotypes, the data scored from the three markers were compiled and analyzed using the Dice similarity coefficient. The genetic similarity matrices based on the Dice coefficients was illustrated in Table 4. The Genetic Similarity (GS) was ranged between 3 and $98.5 \%$. The highest GS value was 98.5\% between Dutch Tulips-1 and Dutch Tulips-2 while the lowest was $3 \%$ between Balady Rose- 1 and Dutch Rose-1. The highest GS values between TaifRoses and other rose genotypes were 92.3, 74.1 and $65.7 \%$ between the three genotypes Hada, Shafa-1, Shafa-2 and Gory rose-Syrian, respectively. The GS values between Taif-Roses and the Egyptian rose genotypes (Balady-1, 2, 3) were the lowest and ranged between 7.4 to $31 \%$. 
Salah El-Din El-Assal et al. / American Journal of Biochemistry and Biotechnology 10 (1): 88-98, 2014

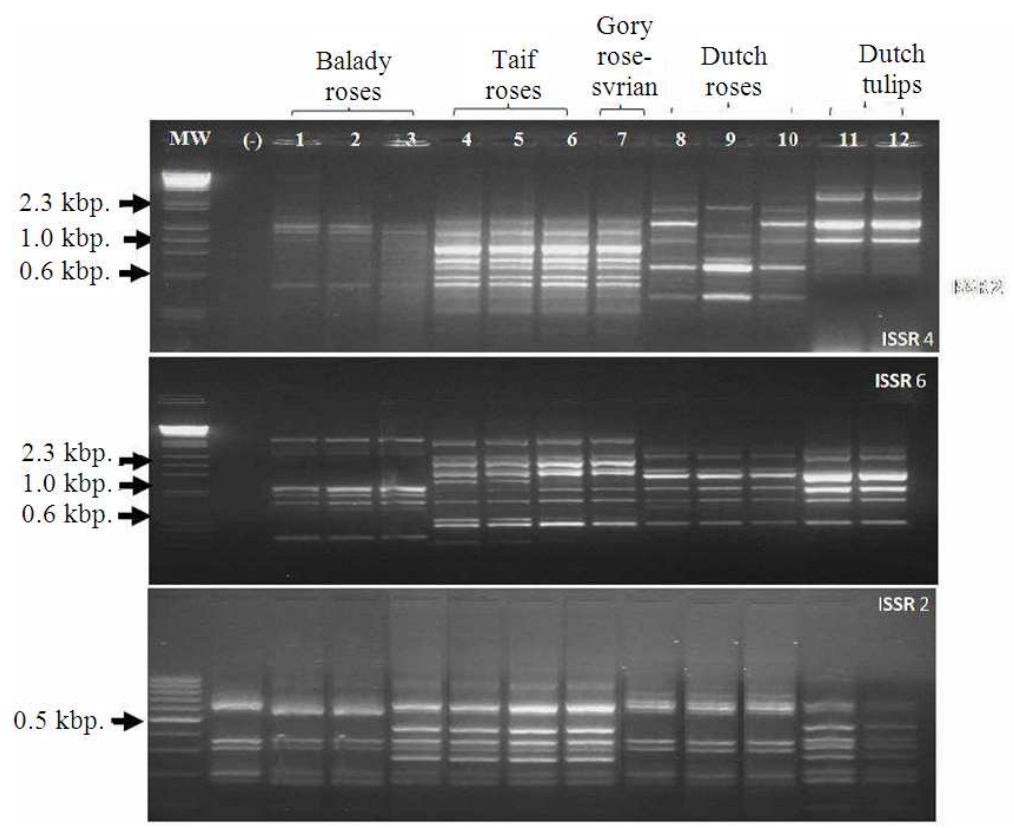

Fig. 2. ISSR profiles of 12 different rose collections using the ISSR primers 2,4 and 6. (1-3) Balady rose1, 2 and 3; (4-6) Taif rose (Hada, Shafa 1 and Shafa 2; (7) Gory rose-Syrian; (8-10) Dutch rose1, 2 and 3 and (11-12) Dutch Tulips 1 and 2. MW: is 1 kb. Ladder for ISSR 4 and 6 and is 100 bp. Ladder for ISSR 2; (-) is a negative PCR sample

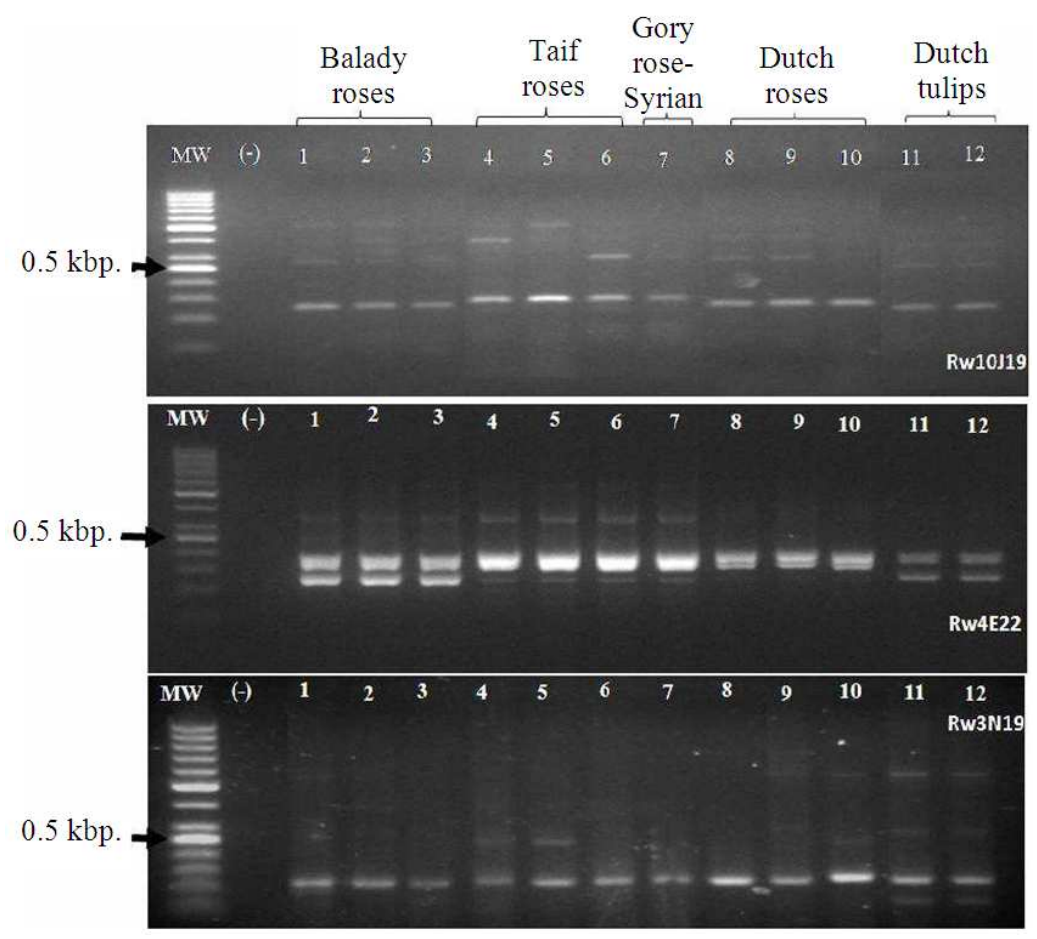

Fig. 3. SSR fingerprinting of 12 different rose collections using three SSR markers. (1-3) Balady rose1, 2 and 3; (4-6) Taif rose (Hada, Shafa 1 and Shafa 2; (7) Gory rose-Syrian; (8-10) Dutch rose1, 2 and 3 and (11-12) Dutch Tulips 1 and 2. MW: is 100 bp.; (-is a negative PCR sample 


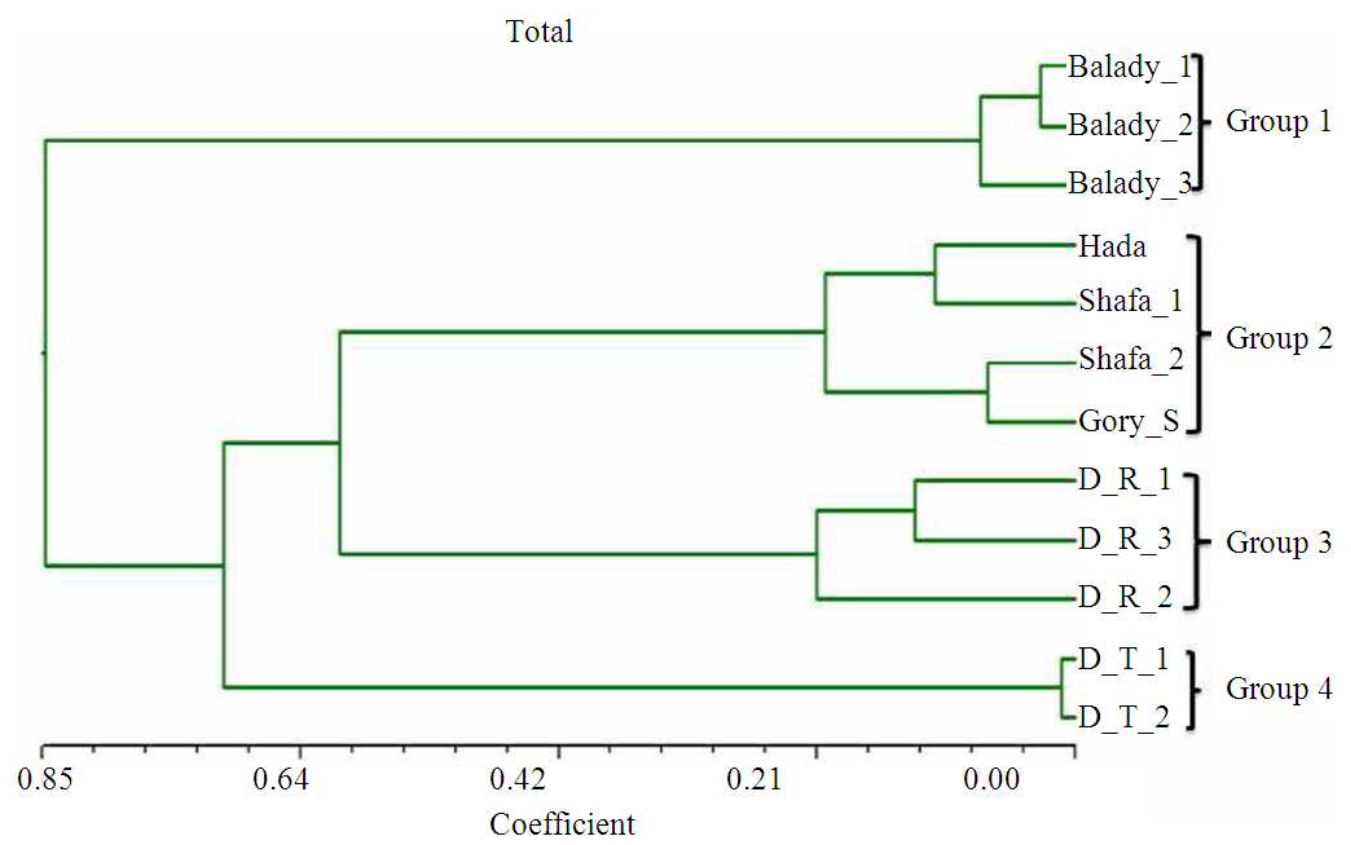

Fig. 4. RAPD's, ISSR's and SSR, s phylogenetic analysis among 12 rose collections. Dutch rose 1, 2, 3; Balady rose 1, 2, 3; Taif rose-Hada; Taif rose-Shafa 1, 2; Gody rose-Syrian and Dutch tulip 1, 2

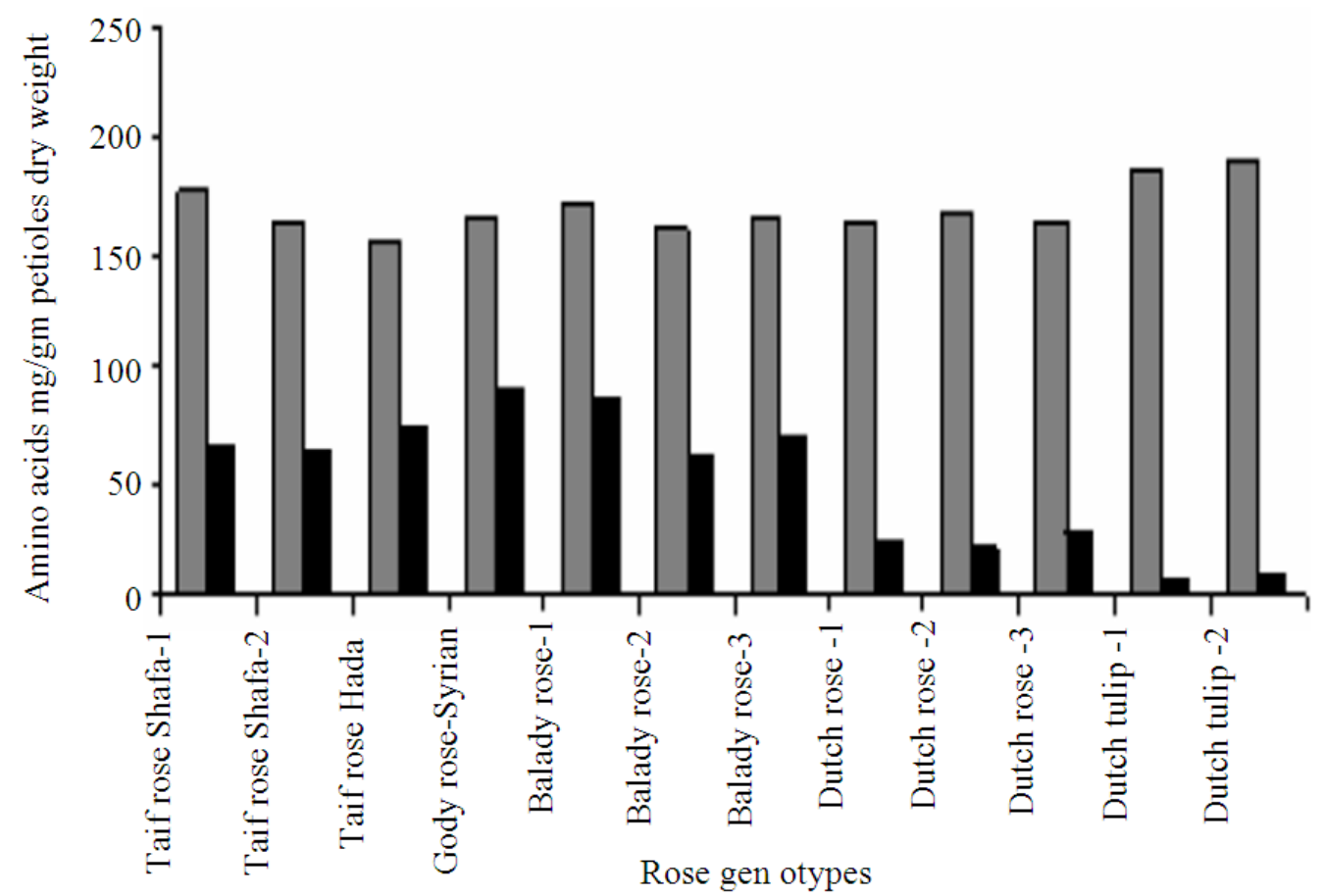

Fig. 5. Amino acid contents analysis using HPLC technology of twelve rose genotypes. Dutch rose 1, 2, 3; Balady rose 1, 2, 3; Taif rose-Hada; Taif rose-Shafa 1, 2; Gody rose-Syrian and Dutch tulip 1,2. Gray bars are presenting the total amino acids in $\mathrm{mg} / \mathrm{gm}$ of rose petioles. Black bars are presenting the aromatic amino acids in $\mathrm{mg} / \mathrm{gm}$ of rose petioles 
Table 4. Genetic dissimilarity (GS) matrices computed according to Dice coefficient from the combined RAPD's, ISSR's and SSR's markers of the twelve rose genotypes, Taif rose-Hada; Taif rose-Shafa 1, 2; Dutch rose 1, 2, 3; Gody roseSyrian and Dutch tulip 1, 2

\begin{tabular}{|c|c|c|c|c|c|c|c|c|c|c|c|c|}
\hline & Bala.-1 & Bala.-2 & Bala. -3 & Hada & Shafa-1 & Shafa-2 & Gory-S & Du-R1 & Du-R2 & Du-R3 & Du-T1 & Du-T2 \\
\hline Bala.1 & 0.000 & & & & & & & & & & & \\
\hline Bala.-2 & 0.0270 & 0.000 & & & & & & & & & & \\
\hline Bala.-3 & 0.0892 & 0.1162 & 0.000 & & & & & & & & & \\
\hline Hada & 0.9237 & 0.9268 & 0.9211 & 0.000 & & & & & & & & \\
\hline Shafa-1 & 0.6931 & 0.7202 & 0.7824 & 0.3466 & 0.000 & & & & & & & \\
\hline Shafa-2 & 0.6931 & 0.7202 & 0.9159 & 0.1234 & 0.1823 & 0.000 & & & & & & \\
\hline Gory-S & 0.6649 & 0.6919 & 0.6363 & 0.3436 & 0.0771 & 0.2594 & 0.000 & & & & & \\
\hline Du-R1 & 0.9721 & 0.9542 & 0.8350 & 0.8047 & 0.8636 & 0.7458 & 0.6222 & 0.000 & & & & \\
\hline Du-R2 & 0.8880 & 0.9150 & 0.7416 & 0.7113 & 0.7702 & 0.7702 & 0.6466 & 0.2475 & 0.000 & & & \\
\hline Du-R-3 & 0.7458 & 0.7729 & 0.7172 & 0.8047 & 0.7458 & 0.7458 & 0.7175 & 0.2231 & 0.1298 & 0.000 & & \\
\hline Du-T1 & 0.9159 & 0.8094 & 0.8873 & 0.8570 & 0.6646 & 0.6646 & 0.7416 & 0.9686 & 0.9371 & 0.9427 & 0.000 & \\
\hline Du-T2 & 0.9277 & 0.9126 & 0.9311 & 0.8267 & 0.7520 & 0.7520 & 0.8291 & 0.9324 & 0.9491 & 0.9447 & 0.0103 & 0.000 \\
\hline
\end{tabular}

While, the genetic similarity values between Taif-Roses Hada, Shafa-1, Shafa-2 and the Dutch roses and Dutch Tulips genotypes (Dutch Rose 1, 2, 3 and Dutch tulips 1, 2) were ranged between 14 and $34 \%$.

UPGMA clustering dendrogram based on DICE similarity index was obtained (Fig. 4). The dendrogram cluster diagram classified the evaluated genotypes in two major clusters and that formed four clear groups. The first cluster contains the three Egyptian Rose genotypes (Balady1, 2 and 3) and represented the first group. The second main cluster was divided into two subclusters in which the first contained the two Dutch Tulips and represented Group 4. The second subcluster was further divided into two branches, the first contained the three Dutch rose genotypes and formed Group 3 and the second contained the three Taif-roses genotypes Hada, Shafa1 and Shafa-2 in addition to the Gory rose-Syrian and formed Group 2.

\subsection{Amino Acid Analysis of Rose Genotypes using High-Performance Liquid Chromatography (HPLC) Technique}

The concentration of the total amino acids and the aromatic amino acids were measured and compared among the twelve rose genotypes (Fig. 5). While, no significant difference in the amount of total amino acid of the twelve genotypes was shown. The ration of the amount of aromatic amino acids to the amount of total amino acid in the three genotypes Balady rose 1, Gody rose-Syrian and Taif rose-Hada was the highest comparing to the rest of the tested genotypes. While, Dutch rose 1, 2, 3 and Dutch tulip 1, 2 genotypes were showed the lowest ratio.

\subsection{In vitro Propagation of Taif Rose}

After four weeks of culture, nodal segment explants showed different responses in production and development of multiple shoots when cultured on Murashige and Skoog medium with different concentrations of 2,4, D and kiniten. Among the various phytohormones concentrations and combinations used in this study, a combination of $1 \mathrm{mg} \mathrm{L}^{-1} 2,4, \mathrm{D}$ and $1 \mathrm{mg} \mathrm{L}^{-1}$ kinetin was found to be the best to yield the highest frequency of proliferation of axillary buds and formation of axillary shoots. While the concentration of $1 \mathrm{mg} \mathrm{L}^{-1}$ of BAP was found to be the best to yield the highest number of multiple shoots (Fig. 1c). The maximum length of shoots was also obtained using the same concentration. The multiple shoots were obtained after 2 months and the highest number of multiple shoots per explant recorded was 2.7 .

\section{DISCUSSION}

\subsection{Molecular Characterization}

The use of molecular markers was aiming to show fast and reliable discrimination of genetic relations of Taif-Roses with other rose genotypes. The three molecular markers have confirmed each other and the combined results were realistic. The discriminating power of the three markers has led to efficient grouping of the 12 rose genotypes and successfully has reveled the genetic relations of Taif-Roses with other rose genotypes using Unweighted Pair Group method (UPGMA). The RAPD's, ISSR's and SSR's have confirmed each other and the combined results were realistic, when the dendrogram clearly separates the analyzed rose genotypes into four major groups. The 
separation of the groups was mainly in correlation to the country of origin and rose-type. The three Taif roses showed highest GS with the Syrian-Gory rose and they were clustered together in the group 2 that further divided into two subclusters. Taif-roses genotypes Hada and Shafa-2 were existed together in the first subcluster with GS exceeded $88 \%$. While, the second sub-cluster contained Shafa-1 and the Gory RoseSyrian with GS of $92.3 \%$.

Since rose is one of the most important ornamental and aromatic crops and represents a major commodity in the commercial market of the floriculture as well as the essential oil industry, the aromatic amino acids measurements was important in order to characterize the different rose genotypes and to select the best genotypes for the commercial usage. Among the 12 tested genotypes, the ration of the amount of aromatic amino acids to the amount of total amino acid in the three genotypes Balady rose 1, Gody rose-Syrian and Taif rose-Hada was the highest. These results may be confirmed the closed genetics relations between Gody rose-Syrian and Taif rose-Hada where these two genotypes were falling in the same cluster (Fig. 4). However, the comparison using the amount of each araomatic amino acid could give better indications than the using of total aromatic amino acids.
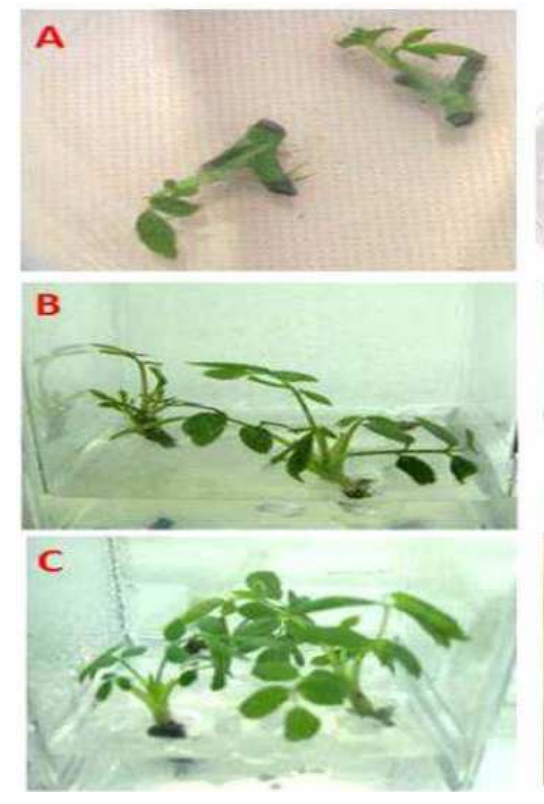

Together, these results indicate that the Taif-roses have closed genetic relations to the Gory rose-Syrian that are cultivating in Syria.

Additionally, a simple protocol for mass production of the Taif-rose genotype Hada using the nodal explants was developed (Fig. 6). Horn (1992) reported that the explants with vegetative meristems are often suitable for enhanced axillary branching and the performance of nodal segments is much better than the shoot tips. The most commonly used explants in shoot proliferation of roses are the nodal stem segments, wherein the axillary bud is made to proliferate and form multiple shoots (Khosravi et al., 2007). It was found that the combination of the auxin 2,4, D and the cytokinin, with the concentrations of $1 \mathrm{mg} \mathrm{L}^{-1}$ for each resulted in the best frequency of proliferation of axillary buds and formation of axillary shoots. Caboni and Tonelli (1999; George, 1993) explained that the presence of auxin in defined combinations with cytokinins in the culture medium is necessary to obtain adventitious shoot formation and the optimum rates of shoot initiation generally occur with combinations of auxin and cytokinin. In addition, the concentration of $1 \mathrm{mg} \mathrm{L}^{-1}$ of BAP was found to be the best to yield the highest number of multiple shoots (Fig. 1c), the maximum length of shoots was also obtained using the same concentration.

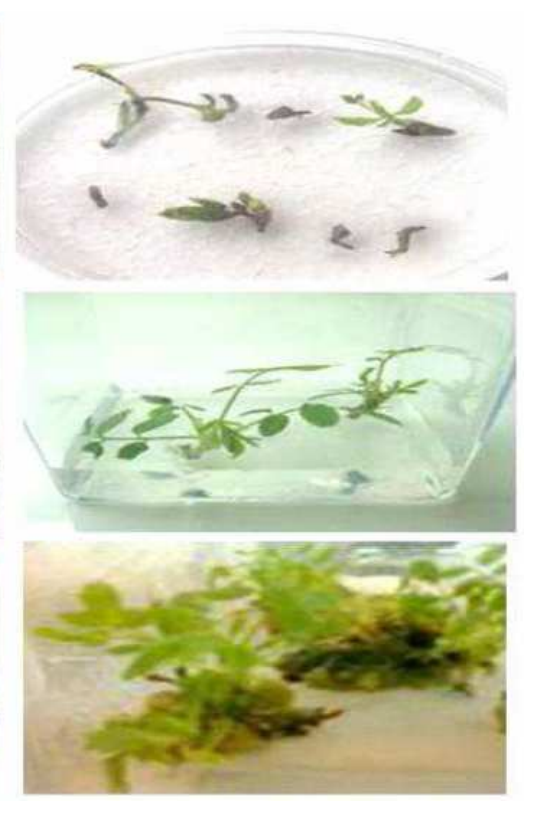

Fig. 6. Micropropagation stages of Taif rose using nodal segments. A: is proliferation of axillary buds and formation of axillary shoots after 3-4 weeks on culture medium. B: is shoots elongation stage ( 2 months) and C: is the multiplication stage (2 months). Each treatment was repeated three times 
Although several different cytokinins have been used in rose proliferation, the best proliferation rate was obtained by using BA. Thi et al. (2008) demonstrated that the most suitable concentration for shoot multiplication was found on MS medium supplemented with $3 \mathrm{mg} \mathrm{L}^{-1}$ BAP.

The long term objective of this work was to use these fingerprints to identify molecular markers that cosegregate and could be used in isolating gene(s) which controlling some important traits. Moreover, the developed In vitro propagation system for Taif-roses could be used for mass production of this economically important crop at any time in the year. Moreover, the improved system can help in the improvement of several desirable traits in Taif-roses such as oil content, increase of flower yield, plant vigour and resistance to fungal diseases through genetic engineering.

\section{REFERENCES}

Atienza, S.G., A.M. Torres, T. Millan and J.I. Cubero, 2005. Genetic diversity in Rosa as revealed by RAPDs. Agric. Conspectus Sci., 70: 75-85.

Baydar, N.G., H. Baydar and T. Debener, 2004. Analysis of genetic relationships among Rosa damascena plants grown in Turkey by using AFLP and microsatellite markers. J. Biotechnol., 111: 263-267. DOI: 10.1016/j.jbiotec.2004.04.014

Bazaid, S.A., 2004. Protein and DNA fragments variation in relation to low temperature in four Rosa hybirida cultivars in Taif, Saudi Arabia. J. Egypt. Acad. Dev., 5: 77-90.

Brown, N., S. Venkatasamy, G. Khittoo, T. Bahorun and S. Jawaheer, 2009. Evaluation of genetic diversity between 27 banana cultivars (Musa spp.) in Mauritius using RAPD markers. Afr. J. Biotechnol., 8: 1834-1840.

Caboni, E. and M.G. Tonelli, 1999. Effect of 1.2benzisoxazole-3-acetic acid on adventitious shoot regeneration and in vitro rooting in apple. Plant Cell Rep., 18: 985-988.

Doyle, J.J. and L.H. Doyle, 1988. Isolation of plant DNA from fresh tissue. Focus, 12: 13-15.

Esselink, G., M. Smulders and B. Vosman, 2003. Identification of cut rose (Rosa hybrida) and rootstock varieties using robust sequence tagged microsatellite site markers. Theor. Applied Genet., 106: 277-286. PMID: 12582853

Gaafar, R.M. and M.M. Saker, 2006. Monitoring of cultivars identity and genetic stability in strawberry varieties grown in Egypt. World J. Agric. Sci., 2: 29-36.
George, E.F., 1993. Plant Propagation by Tissue Culture. 2nd Edn., Part 1. The Technology. Exegetics Ltd, Basingstoke, UK.

Gupta, P.K. and R.K. Varshney, 2000. The development and use of microsatellite markers for genetic analysis and plant breeding with emphasis on bread wheat. Euphytica, 113: 163-185. DOI: 10.1023/A:1003910819967

Helsen, M.M., R.M. Van den Broeke, R.S.W. Van Der Wal, W.J. Van de Berg and E. van Meijgaard et al., 2008. Elevation changes in Antarctica mainly determined by accumulation variability. Science, 320: 1626-1629. DOI: 10.1126/science.1153894

Horn, W.A.H., 1992. Micropropagation of Rose (Rosa L.). In: Biotechnology in Agriculture and Forestry, Bajaj, Y.P.S. (Ed.), Springer-Verlag, Berlin.

Jabbarzadeh, Z., M. Khosh-khui, H. Salehi and A. Saberivand, 2010. Inter Simple Sequence Repeat (ISSR) markers as reproducible and specific tools for genetic diversity analysis of rose species. Afr. J. Biotechnol., 9: 6091-6095. DOI: 10.5897/AJB10.133

Kaur, N., R.K. Sharma, D. Dhyani, S. Karthigeyan and P.S. Ahuja, 2007. Molecular characterization of interspecific hybrids of scented roses using RAPD markers. Acta Horticulturae, 751: 175-179.

Khosravi, H. and E. Kabir, 2007. Introducing a very large dataset of handwritten Farsi digits and a study on their varieties. Patt. Recognit. Lett., 28: 11331141. DOI: 10.1016/j.patrec.2006.12.022

Maxwell, S.E. and H.D. Delaney, 1989. Designing Experiments and Analyzing Data. 1st Edn., Wadsworth, Belmont, CA, pp: 260.

Murashige, T. and F. Skoog, 1962. A revised medium for rapid growth and bio assays with tobacco tissue cultures. Physiol. Plantarum, 15: 473-497. DOI: 10.1111/j.1399-3054.1962.tb08052.x

Nowbuth, P., G. Khittoo, T. Bahorun and S. Venkatasamy, 2005. Assessing genetic diversity of some Anthurium andraeanum Hort. cut-flower cultivars using RAPD Markers. Afr. J. Biotechnol., 4: 1189-1194.

Rajapakse, S., D.H. Byrne, L. Zhang, N. Anderson and K. Arumuganathan et al., 2001. Two genetic linkage maps of tetraploid roses. Theor. Applied Genet., 103: 575-583. DOI: 10.1007/PL00002912

Rholf, F.J., 1990. NTSYS-pc Numerical Taxonomy and Multivariate Analysis System Version 1.8. Applied Biostatist. 
Rout, G., R. Kullu, J. Senapati, S.K. Aparajita and A. Mohapatra, 2007. Identification and genetic relationship among Polyscias and Schefflera (Araliaceae) using RAPD and ISSR markers. Plant Biotechnol., 24: 519-525.

Sesli, M. and E.D. Yeğenoğlu, 2009. Genetic analysis on wild olives by using RAPD markers. Afr. J. Agric. Res., 4: 707-712.

Thi, K.O., A.A. Khai and K.M. Lwin, 2008. Micropropagation of Rose (Hybrid Rosa spp.) by in vitro culture technique. Proceedings of the International Conference on Sustainable Development: Issues and Prospects for the GMS, Nov. 12-14.

Williams, J.G.K., A.R. Kubelik, K.J. Livak, J.A. Rafalski and S.V. Tingey, 1990. DNA polymorphisms amplified by arbitrary primers are useful as genetic markers. Nucleic Acids Res., 18: 6531-6535. PMID: 1979162
Yamanaka, K., S. Horimoto, M. Matsuoka and K. Banno, 1994. Analysis of thiamine in dried yeast by high-performance liquid chromatography and high-performance liquid chromatography/atmospheric pressure chemical ionization-mass spectrometry. Chromatographia, 39: 91-96. DOI: 10.1007/BF02320465

Zhang, L.H., D.H. Byrne, R.E. Ballard and S. Rajapakse, 2006. Microsatellite marker development in rose and its application in tetaploid mapping. J. Am. Soc. Hort. Sci., 131: 380-387. 